\title{
Solution of series-parallel photovoltaic arrays model using global optimization algorithms
}

\author{
Solución de un modelo de arreglos fotovoltaicos serie-paralelo utilizando \\ algoritmos de optimización global
}

\author{
J. D. Bastidas-Rodríguez (iD, J. M. Cruz-Duarte iD, C. R. Correa-Cely iD
}

\begin{abstract}
Models of series-parallel (SP) photovoltaic (PV) arrays focus on the system of nonlinear equations that represents the array's electrical behavior. The solution of the system of nonlinear equations can be posed as an optimization problem and solved with different methods; however, the models do not formulate the optimization problem and do not evaluate different optimization algorithms for its solution. This paper proposes a solution, using global optimization algorithms, of the mathematical model that describes the electrical behavior of a SP generator, operating under uniform and partial shading conditions. Such a model is constructed by dividing the generator into strings and representing each module in the string with the single-diode model. Consequently, for each string a system of nonlinear equations is build applying the Kirchhoff's laws, where the unknowns are the modules' voltages. The solution of the resulting nonlinear equation system is posed as an optimization problem, where the objective function is defined as the sum of the squared of each nonlinear equation. Minimum and maximum values of each voltage are defined from the datasheet information of the modules and bypass diodes. As a demonstrative example, we arbitrarily select two well-known algorithms to solve this problem: Genetic Algorithms and Particle Swarm Optimization. Simulation results show that both algorithms solve the optimization problem and allow the reproduction of the generator's characteristic curves. Moreover, the results also indicate that the optimization problem is correctly defined, which opens the possibility explore other optimization algorithms to reduce the computation time.
\end{abstract}

Index Terms-Global optimization; Partial Shading; Photovoltaic Arrays; Series-Parallel.

This manuscript was sent on August 13, 2019 and accepted on March 11, 2020 .

J. D. Bastidas-Rodríguez is with the Departamento de Ingeniería Eléctrica, Electrónica y Computación, Universidad Nacional de Colombia, Carrera 27 \# 64-60, Manizales, Colombia (e-mail: jubastidasr@unal.edu.co).

J. M. Cruz-Duarte is with the Grupo de Investigación con Enfoque Estratégico en Sistemas Inteligentes, Escuela de Ingeniería y Ciencias, Tecnológico de Monterrey, Av. Eugenio Garza Sada 2501 Sur, Col. Tecnológico, Monterrey, México (e-mail: jorge.cruz@tec.mx).

C. R. Correa-Cely is with the Escuela de Ingenierías Eléctrica, Electrónica y de Telecomunicaciones, Universidad Industrial de Santander, Carrera 27 calle 9, Bucaramanga, Colombia (e-mail: crcorrea@ saber.uis.edu.co).
Resumen-Los modelos de arreglos fotovoltaicos (FV) en serieparalelo (SP) se enfocan en el sistema de ecuaciones no lineales que represental comportamiento eléctrico del arreglo. La solución del sitemas de ecuaciones se puede plantear como un problema de optimización y resolverse con diferentes métodos; sin embargo, los modelos no formulan el problema de optimización y no evaluan diferentes algoritmos de optimización para su solución. Este artículo propone una solución, utilizando algoritmos de optimización global, del modelo matemático que describe el comportamiento eléctrico de un generador fotovoltaico en serie-paralelo, que opera bajo condiciones uniformes y de sombreados parciales. Dicho modelo se construye dividiendo el generador en cadenas y representando cada módulo en la cadena con el modelo de diodo-único. En consecuencia, para cada cadena se construye un sistema de ecuaciones no lineales aplicando las leyes de Kirchhoff, en donde las incógnitas son los voltajes de los módulos. La solución del sistema de ecuaciones no lineales resultante se plantea como un problema de optimización, donde la función objetivo se define como la suma del cuadrado de cada ecuación no lineal. Los valores mínimos y máximos de cada voltaje se definen a partir de la información de la hoja de datos de los módulos y de los diodos de derivación. Como ejemplo demostrativo, se seleccionaron arbitrariamente dos algoritmos bien conocidos para resolver este problema: Algoritmos Genéticos y Optimización por Enjambre de Partículas. Los resultados de simulación muestran que los dos algoritmos ambos algoritmos resuelven el problema de optimización y permiten la reproducción de las curvas características del generador. Adicionalmente, los resultados también indican que el problema de optimización se definió correctamente, lo cual abre la posibilidad de explorar otros algoritmos de optimización para reducir el tiempo de cómputo.

Palabras claves-Arreglos Fotovoltaicos; Optimización Global; Serie-Paralelo, Sombreado Parcial.

\section{INTRODUCTION}

P HOTOVOLTAIC (PV) generators have established as one of the most important renewable energy source, since the energy source is available almost everywhere, is free and inexhaustible. Moreover, PV generators do not emit greenhouse gases, do not have mobile parts and are modular, which allows implementing PV generators from a few watts to MW [1], [2]. These and other advantages have contributed to 
the continuous increment of the installed PV capacity. Just in 2017, $98 \mathrm{GW}$ were installed around the world to reach a global installed capacity of $402 \mathrm{GW}$, approximately [3], [4]. PV generators transform the sunlight energy into electrical power through PV cells. These cells are connected in series to form modules, which are characterized by a protection diode named bypass diode. In turn, one or more modules connected in series form a PV panel, which is the commercial unit used to form the PV generators. A PV generator is typically formed by PV panels connected in series to form strings, in order to reach the voltage levels required by a particular application. Finally, two or more strings are connected in parallel to supply the power required by the load [1], [2]. This particular arrangement of PV panels is denominated Series-Parallel (SP) configuration and it is the most widely adopted in different applications [1], [2].

When all the modules in the PV generator are the same and they operate under the same conditions, i.e., all of them receive the same irradiance and their cells have the same temperature, it is said that the PV generators operates in uniform or homogeneous conditions [1], [2], [5]. In these conditions the bypass diodes of the PV modules are inactive; therefore, the current vs. voltage (I-V) has a single knee, which produces a single Maximum Power Point (MPP) in the power vs. voltage $(\mathrm{P}-\mathrm{V})$ curve. However, in real applications the PV modules in a generator may operate under different irradiance and temperature conditions due to the shadow produced by surrounding objects (like trees, poles, buildings, etc.), soiling of some modules, aging, or manufacturing tolerances in the cells of the modules. These operating conditions are denominated non-homogeneous or mismatching conditions and they may significantly reduce the power produced by a PV generator [2], [6], [7]. The maximum current of a PV module, i.e., the short-circuit current $\left(I_{s c}\right)$, is proportional to the effective irradiance on the module surface. Hence, the short-circuit current of a PV module operating under mismatching conditions $\left(I_{s c, m}\right)$ is less than the shortcircuit current of a PV module under uniform conditions $\left(I_{s c, u}\right)$. In a string, a mismatched PV module may be connected in series with other modules operating in uniform conditions. When the string current is less than $I_{s c, u}$ and $I_{s c, m}$, the bypass diodes of all the modules are inactive and all the modules deliver power. Nevertheless, when the string current is greater than the $I_{s c, m}$ and less than $I_{s c, u}$, the bypass diode of the mismatched module is active to allow the flow of the difference between the string current and $I_{s c, m}$. When a bypass diode is active, the PV module does not deliver power to the string and the voltage across its terminals is approximately zero [2], [6], [7]. In general, the activation or deactivation of the bypass diodes in a PV generator depend on its operating point (current and voltage) and the mismatching conditions of the PV modules. Such activation and deactivation of the bypass diodes produce multiple knees in the generator's I-V curve and, as consequence, multiple MPPs in the generator's P-V curve, where one of them is the global MPP (GMPP) and the rest are local MPPs (LMPPs) [2], [6], [7].

Accurate mathematical models of PV arrays operating under uniform and mismatching are important for different applications in PV systems. They can be used to estimate the power and energy produced by a PV generator, to perform realistic economic analysis $\mathrm{PV}$ systems, to evaluate the performance of MPP tracking (MPPT) techniques, to propose model based reconfiguration techniques, to implement PV array emulators and other applications of PV systems [2], [8]. A model of a PV array, under uniform or mismatching conditions, needs to calculate the array current for a given voltage, since the array voltage is fixed by the power converter to which it is connected. From this calculation it is possible to reproduce the generator's I-V and P-V curves or to perform dynamic simulations of the PV array [2]. In the literature there are different models of SP arrays, which are focused on the procedure to define the set of nonlinear equations to represent the strings and arrays [9]-[14]. However, these methods do not analyze the details of the numerical methods or the optimization strategies to solve those systems of nonlinear equations. In SP configurations, each string can be analyzed independently because all of them are connected in parallel, hence, the strings' voltages are the same and correspond to the array voltage [9]-[14]. Some authors have defined a system of nonlinear equations by applying the Kirchhoff's current law in each node of the string [9], [10]. Therefore, a string with $N$ modules and one blocking diode is described by a system of $N+1$ nonlinear equations. Reference [9] has introduced an explicit description of the Jacobian matrix associated to each string and mention that it needs to be inverted to solve the system of nonlinear equations by using Newton-Raphson method. Nevertheless, the authors have not mentioned the numerical method used to solve the nonlinear equation system. The inverse of the Jacobian matrix associated to a string can be explicitly defined by using the Schür complement, as introduced by [11]. Such a definition reduces the calculation burden of the Newton-Raphson method and the simulation time. However, such a method requires a guess solution sufficiently close to the real one in order to solve the system of nonlinear equations. This condition is difficult to fulfill in a PV string due to the voltages of a PV module vary depending on the string operating point (i.e., string voltage). A solution for this problem is presented by [11], where the authors use a damped Newton-Raphson method and provide complex procedure to define the guess solution. Research of [10] proposed a modeling procedure that reduces the complexity of the nonlinear equation system of a string. On the one hand, they represented each PV module by its ideal representation, i.e., disregarding the ohmic losses (series resistance) and leakage currents (parallel resistance). On the other hand, the authors implemented a method to calculate the inflection points in the string I-V curve, which are associated with the activation and deactivation of the bypass diodes in the modules. Then, it was possible to reduce the number of equations that represent a string because the modules with active bypass diodes are neglected. Although [10] proposed an explicit definition of the Jacobian matrix associated with the reduced system of nonlinear equations, they used NewtonRaphson method to find the solution and they do not provide guidelines or a procedure to define the guess solution. Authors of [12] presented a system of nonlinear equations to model SP arrays, as well as other configurations, formed by six panels with two modules each. The authors defined a fixed set of nonlinear equations for each configuration by using the 
Kirchhoff's current and voltage laws. Such a system of nonlinear equations is solved by Trust-Region optimization method. However, the authors did not define the optimization problem, and they did neither justify the selection of this solution method nor analyze other optimization methods. Nevertheless, there are other models for SP array available in literature [13], [14] focused on reproducing the I-V and P-V curves of the array. But those models did not pose a system of nonlinear equations that represents the electrical behavior of an SP array and, as a result, they do not require solving a system of nonlinear equations. Instead, they calculated the I-V curve of each module and constructed the I-V curve of the array by operating (i.e., adding or multiplying) the modules' curves, which is not practical if the operating conditions are continuously changing.

This paper introduces a numerical alternative to solve the nonlinear system of equations that describes the electrical behavior of a SP array. The solution of such a system of equations is posed as an optimization problem, and it is solved by using two widely used global optimization algorithms, i.e., Genetic Algorithm (GA) and Particle Swarm Optimization (PSO). The performance of the algorithms was evaluated for such arrays, where the Root Mean Squared Error (RMSE) of the solutions were compared with the electrical implementation of a SP array in multiple tests. Moreover, the effectiveness of the algorithms to solve a small and a large array is evaluated by using number of evaluations of the objective function in multiple runs of the Global Optimization Algorithms (GOAs). The rest of the paper is organized as follows, Section 2.1 introduces the model of an SP array, Sections 2.2 details the mathematical foundations of the optimization problem, Section 2.3 presents the global optimization algorithms used to solve the problem, Section 2.4 introduces the methodology, Section 3 contains the results and Section 4 closes the paper with the conclusions.

\section{MATERIALS AND METHODS}

This section introduces the model of Series-Parallel PV arrays, the mathematical foundations to transform the model solution into an optimization problem and the definition of the optimization problem proposed in this paper. Moreover, this section also includes the general descriptions of the two optimization algorithms used.

\section{A. Model of series-parallel photovoltaic arrays}

An SP array is formed by one or more strings connected in parallel, as shown in Fig. 1. Thus, each string is formed by two or more modules connected in series and a blocking diode to avoid inverse current through the string. Moreover, the number of modules in a string depends on the voltage required by the load and the number of strings depends on the power to be supplied.

All the strings in an SP array are connected in parallel, therefore, they can be analyzed independently because each one forms an electric loop with the array voltage. Such a condition implies that each string can be represented by a system of nonlinear equations, where the unknowns are voltages of the modules and the blocking diode, and the array voltage is considered known because it is fixed by the power converter where the PV array is connected to. Then, the string current can be simply calculated by using the voltage of one module in the string and the array current is calculated by adding the currents of all the strings.

The following subsections describe the electrical model of each PV module and the system of nonlinear equations of one string as well as the calculation of the string and array currents.

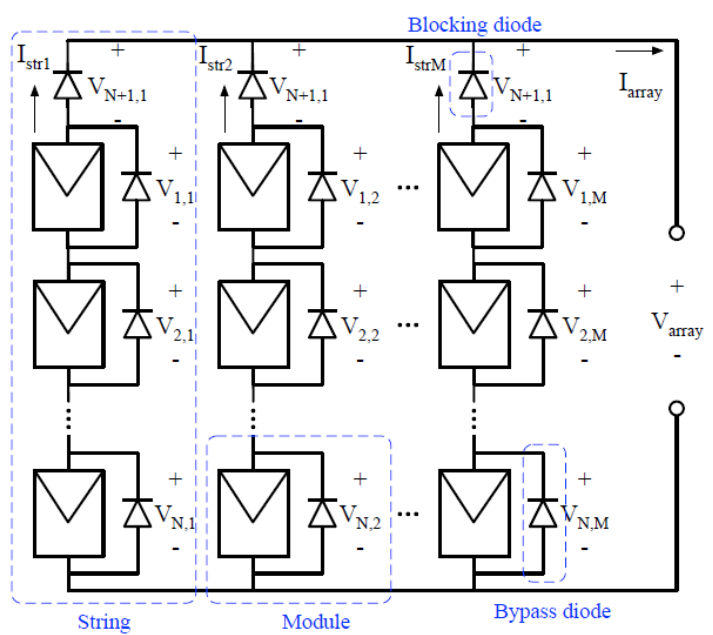

Fig. 1. Definition of a PV array in Series-Parallel configuration with M strings and $\mathrm{N}$ modules per string.

\section{1) Electrical model of a PV module}

A PV module is composed by Ns PV cells connected in series with a bypass diode (BD) connected in parallel to protect the cells, as shown in Fig. 2 (left); where the current source $\left(I_{p h}\right)$ represents the current generated by the photovoltaic effect, the diode $(D)$ introduces the nonlinear behavior of the $P N$ junctions of the PV cells, resistance $R_{h}$ describes the leakage current between the $P N$ junction, and $R_{S}$ includes the ohmic losses produced by the semiconductormetal contacts and the cables. In literature, there are other electrical models of a PV modules [2]. Nevertheless, the single-diode model (SDM) (see Fig. 2) is the most widely adopted because it provides a trade-off between complexity and accuracy.

Applying the Kirchhoff current law (KCL) at one terminal of the PV module it is possible to obtain the module output current $(I)$ from the currents through $R_{S}\left(I_{R s}\right)$ and diode BD $\left(I_{b d}\right)$, as shown in (1). In turn, $I_{R s}$ is defined in terms of $I_{p h}$ and the currents through diode $D\left(I_{d}\right)$ and $R_{h}\left(I_{R h}\right)$, as described by (2). Currents $I_{d}, I_{R h}$ and $I_{b d}$ depend on the module voltage $(V)$ as introduced in (3), (4) and (5), respectively, where $I_{s a t}$ and $I_{s a t, b d}$ are the inverse saturation current of diodes $D$ and $B D$, respectively, and $V_{t, d}$ and $V_{t, b d}$ are proportional to the thermal voltages of diodes $D$ and $B D$, respectively.

The last two parameters are defined as follows: $V_{t, d}=$ $N_{s} \eta k T / q$ and $V_{t, b d}=\eta_{b d} k T / q$, where $\eta$ and $\eta_{b d}$ are the ideality factors of diodes $D$ and $B D$, respectively, $k$ is the Boltzmann constant, $q$ is the electron charge, and $T$ is the 
temperature of the PV cells and the bypass diode, which are assumed equal [15], [16].

$$
\begin{gathered}
I=I_{R s}+I_{b d} \\
I_{R s}=I_{p h}-I_{d}+I_{R h} \\
I_{d}=I_{\text {sat }} \cdot\left(\exp \left(\frac{V+\left(I-I_{b d}\right) R_{s}}{V_{t, d}}\right)-1\right) \\
I_{R h}=\frac{V+\left(I-I_{b d}\right) R_{s}}{R_{h}} \\
I_{b d}=I_{s a t, b d} \cdot\left(\exp \left(\frac{-V}{V_{t, b d}}\right)-1\right)
\end{gathered}
$$

Combining (1)-(5), it is possible to obtain a single expression that describes the relation between the module current $(\mathrm{I})$ and voltage $(\mathrm{V})$ in a single expression, as shown in (6), where I and $\mathrm{V}$ are the module variables and $I_{p h}, I_{s a t}, \eta$, $R_{s}, R_{h}, V_{t}, I_{s a t, b d}$ and $\eta_{b d}$ are PV module parameters. On the one hand, the parameters of the Ns PV cells $\left(I_{p h}, I_{s a t}, \eta, R_{s}\right.$ and $R_{h}$ ) can be obtained from the electrical characteristics of the PV module's datasheet, by using some methods reported in literature ([17], [18]), and they vary with the irradiance $(G)$ and $T$. On the other hand, the parameters of the bypass diodes can be estimated from the forward voltage and current, in a given operation point, provided in the bypass diode's datasheet.

$$
\begin{array}{r}
I=I_{p h}-I_{s a t}\left(\exp \left(\frac{V+\left(I-I_{b d}\right) R_{s}}{V_{t, d}}\right)-1\right) \\
-\frac{V+\left(I-I_{b d}\right) R_{s}}{R_{h}}+I_{b d}
\end{array}
$$

It is worth noting that (6) is an implicit and nonlinear function, where $I$ can be solved for a given value of $V$ by using the Lambert $W$ function or numerical methods [9]. Using the Lambert $W$ function $(W)$ it is possible to express $I$ as a function of $V$ as shown in (7), where $I_{b d}$ is defined in (5).

$$
\begin{gathered}
I(V)=\frac{R_{h}\left(I_{p h}+I_{s a t}\right)-V}{R_{s}+R_{h}}-\frac{V_{t, d}}{R_{s}} W(\theta)+I_{b d} \\
\theta=\frac{R_{s} R_{h}}{V_{t, d}\left(R_{s}+R_{h}\right)} I_{s a t}\left(\exp \left(\frac{R_{s} R_{h}\left(I_{p h}+I_{s a t}\right)+V}{V_{t, d}\left(R_{s}+R_{h}\right)}\right)\right)
\end{gathered}
$$

\section{2) Calculation of the string current}

To calculate the current of a string, it is necessary to determine the voltage of all the modules and the blocking diode. Then, string current is calculated by replacing voltage of any module into (6). For a string with $N$ modules and one blocking diode (see Fig. 2), there are $N+1$ unknown voltages, i.e., the voltages from module one $\left(V_{1}\right)$ to module $N\left(V_{N}\right)$ and the voltage of the blocking diode $\left(V_{N+1}\right)$. Therefore, the system of $N+1$ nonlinear equations is obtained by applying the KCL and the Kirchhoff voltage law (KVL). The KCL is applied to each node in the string (see Fig. 2) to obtain $\mathrm{N}$ equations. The last equation comes from the application of KVL to the loop formed by the string and the array voltage $\left(V_{\text {array }}\right)$, which is known.
The system of nonlinear equation $(F)$ is shown in (8), where $\vec{V}$ is the vector formed by the $N+1$ unknown voltages, $I_{i}\left(V_{i}\right)$ is the current of module $i$ as function of its voltage $\left(V_{i}\right)$ according to (7), and $I_{b l k}\left(V_{N+1}\right)$ is the current of the blocking diode as function of its voltage as show in (9). The parameters $I_{s a t, b l k}$ and $V_{t, b l k}$ correspond to the inverse saturation current and thermal voltage of the blocking diode, which can be obtained from the diode's datasheet.

$$
F(V)=\left[\begin{array}{c}
F(1)=I_{1}\left(V_{1}\right)-I_{2}\left(V_{2}\right)=0 \\
F(2)=I_{1}\left(V_{1}\right)-I_{3}\left(V_{3}\right)=0 \\
\vdots \\
F(N-1)=I_{1}\left(V_{1}\right)-I_{N}\left(V_{N}\right)=0 \\
F(N)=I_{1}\left(V_{1}\right)-I_{b l k}\left(V_{N+1}\right)=0 \\
F(N+1)=V_{1}+V_{2}+\cdots+V_{N}+V_{N+1}-V_{\text {array }}
\end{array}\right]
$$

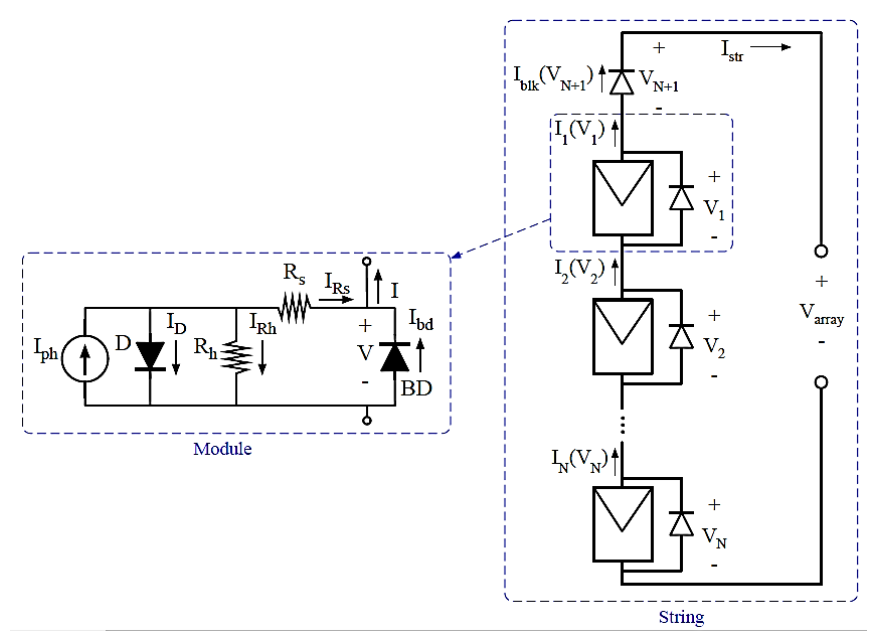

Fig. 2. Single-diode model of a PV module (left). Definition of a string with $N$ modules and one blocking diode (right).

$$
I_{b l k}\left(V_{N+1}\right)=I_{s a t, b l k} \cdot\left(\exp \left(\frac{-V_{N+1}}{V_{t, b l k}}\right)-1\right)
$$

The unknown voltages are obtained by solving $F(\vec{V})$, then, the string current can be calculated by evaluating (7) for any module or evaluating (9) for $V_{N+1}$, because all the modules and the blocking diode are connected in series and, as consequence, they have the same current. In this paper the string current is calculated by evaluating (9), since it is simpler than (7), as shown in (10).

$$
I_{b l k}\left(V_{N+1}\right)=I_{s a t, b l k}\left(\exp \left(\frac{-V_{N+1}}{V_{t, b l k}}\right)-1\right)
$$

\section{3) Calculation of the array current}

Considering a SP array formed by $\mathrm{M}$ parallel connected strings, then the array current is calculated as shown in (11) according to [2], [9], [19]. It is worth noting that the current in a string is independent of the other strings, hence each string current can be calculated independently, which allows the model implementation by using parallel computing [20].

$$
I_{\text {array }}=\sum_{k=1}^{M} I_{s t r, k}
$$




\section{B. Mathematical foundations}

\section{1) Transforming the problem}

In this section, we describe how to transform the solution of a system of nonlinear equations into an optimization problem, which can be solved through global optimization algorithms.

Definition 1 (Real roots finding problem). Let $\mathbb{R}$ be the set of real numbers and let $\mathbb{X}$ be a non-empty subset of $\mathbb{R}^{n}$, say $\mathbb{X} \subset \mathbb{R}^{n}$. Then, let us consider the following system of equations:

$$
\left\{\begin{array}{c}
f_{1}(\vec{x})=0 \\
f_{2}(\vec{x})=0 \\
\vdots \\
f_{m}(\vec{x})=0
\end{array}\right.
$$

since $\vec{x} \in \mathbb{R}^{n}$ and $f_{i}: \mathbb{X} \rightarrow \mathbb{R}, \forall i=1,2, \ldots, m$. Therefore, let the problem be established as: the designing and implementation of an algorithm to find all solutions in the set $\mathbb{X}$ for the system in (12). In other words, an algorithm to find all $\vec{a} \in \mathbb{X}$ such that $f_{i}(\vec{a})=0, \forall i=1,2, \ldots, m$.

\section{2) Minimization}

Definition 2 (Direct image). Let $\mathbb{X}, \mathbb{Y}$ be sets, $\mathbb{A} \subseteq \mathbb{X}$ be a subset, and $f: \mathbb{X} \rightarrow \mathbb{Y}$ be a function. Define the direct image of $\mathbb{A}$ under $f$ as the set $f[\mathbb{A}]:=\{f(\vec{x}): \vec{x} \in \mathbb{A}\}$. Notice that $f[\mathbb{A}] \subseteq \mathbb{Y}$ for all $\mathbb{A} \subseteq \mathbb{X}$.

Theorem 1. Let $\mathbb{X}, \mathbb{Y}$ be sets and $f: \mathbb{X} \rightarrow \mathbb{Y}$ be a function. Suppose $\left\{X_{a}\right\}_{\alpha \in J}$ is a collection of subsets of $\mathbb{X}$. Then,

$$
f\left[\bigcup_{\alpha \in J} \mathbb{X}_{\alpha}\right]=\bigcup_{\alpha \in J} f\left[\mathbb{X}_{\alpha}\right]
$$

where $\cup_{\alpha \in J}$ is the well-known union operator for a finite set collection.

Definition 3 (Minimization). Let $\mathbb{X}, \mathbb{Y}$ be sets, $\vec{a} \in \mathbb{X}$ and $f: \mathbb{X} \rightarrow \mathbb{Y}$ be a function. If $\mathbb{Y}$ is a totally ordered set, then it is said that $f$ attains its minimum along $\mathbb{X}$ in $\vec{a}$ iff $f(\vec{a}) \leq$ $f(\vec{x})$ for all $\vec{x} \in \mathbb{X}$.

\section{3) Equation's systems and optimization}

This subsection shows the theorem about the relationship between a system of equations in real numbers and an optimization problem.

Definition 4 (Sum of squares). Consider the problem stated in Definition 3. Let $f: \mathbb{X} \rightarrow \mathbb{R}_{++}$be a function given by

$$
f(\vec{x}):=\sum_{i=1}^{m}\left[f_{i}(\vec{x})\right]^{2}, \text { with } \vec{x} \in \mathbb{X} .
$$

Notice that $f$ is well-defined, plus its image along $\mathbb{X}$ consists of non-negative real numbers.

Thereupon, given $f(\vec{x}) \geq 0$ for all $\vec{x} \in \mathbb{X}$ and $\mathbb{R}$ is a totally ordered set, there exists the infimum of $f[\mathbb{X}]$ over $\mathbb{R}$, and besides this infimum is non-negative, $\inf \{f[\mathbb{X}]\} \geq 0$. Hence, if exists the minimum of $f$ over $\mathbb{X}$, this minimum must be non-negative. Moreover, if the system in (12) has solution in domain given by $\mathbb{X}$, the minimum of $f$ over $\mathbb{X}$ exists and it is zero; that is proved as follows:

PSEUDOCODE 1. Methodology for finding system's roots using an optimization technique

Input: System of equation (12) and the set $\mathbb{X}$.

Output: Point $\overrightarrow{\boldsymbol{a}} \in \mathbb{X}$.
1: Built $f$ using (14).

2: Minimize $f$ over $\mathbb{X}$.

3: Let $\overrightarrow{\boldsymbol{a}} \in \mathbb{X}$ be a minimum point for $f$.

4: if $\boldsymbol{f}(\overrightarrow{\boldsymbol{a}})=\mathbf{0}$, then $\overrightarrow{\boldsymbol{a}}$ fulfills (12);

else, the system in (12) has no solution in $\mathbb{X}$. end if

\section{Optimization algorithms}

1) Genetic algorithms (GA)

It was formally presented by Holland in 1975 as the canonical algorithm, opening doors to a complete family of computational models inspired in evolutionary processes, such as the natural selection [21]. For a given objective function from an optimization problem, GA searches its solution through three basic operators well-settled in the literature, i.e., inheritance, cross-over, and mutation. The basic idea consists of making each new generation (the off-springs) better than its parents by selecting their most positive features but considering the chance of abruptly modifying (mutating) them [22]. This algorithm has been implemented in a huge number of practical applications since its appearance [23]. There are at least one hundred thousand documents related to Genetic Algorithms in Scopus's database. Pseudocode 2 displays a basic implementation for GA. It utilizes the Stochastic Universal Sampling (SUS) technique as the Selection function for choosing parents [24]. Subsequently, the Elitism, Crossover and Mutation procedures are contemplated for the next generation creation. For the Elitism process, a portion $\left(M_{e} \leq M\right)$ of the total population $M$ survives to the next generation based on its fitness values. For Crossover procedure, a fraction $C_{f} \in[0,1]$ of the remaining next generation is created by combining pairs of parents, $M_{c}=$ $\left[C_{f}\left(M-M_{e}\right)\right]$. The offsprings' final portion $\left(M-M_{e}-M_{c}\right)$ is completed via the Mutation procedure, which consists of randomly perturbing the individuals' solutions [25]. In this work, the conventional scattered crossover function and the Gaussian random distribution are considered for the last mentioned two procedures, respectively.

Pseudocode 2. Genetic Algorithm (GA).

Input: $f: \mathbb{R}^{D} \rightarrow \mathbb{R}, M>2, M_{e} \in(0, M), C_{f} \in[0,1], N \gg 1$, and stopping criteria

Output: $\overrightarrow{\boldsymbol{x}}_{*}^{n}$

1: Initialize $\boldsymbol{x}^{\mathbf{0}}$ as the first generation, and make $\boldsymbol{n} \leftarrow \mathbf{0}$

2: repeat

3: $\quad$ Perform the Selection procedure.

4: $\quad$ Perform the Elitism procedure.

5: $\quad$ Perform the Cross-over procedure.

6: Perform the Mutation procedure.

7: $\quad$ Find $\overrightarrow{\boldsymbol{x}}_{*}^{n+1}$ and Make $\boldsymbol{n} \leftarrow \boldsymbol{n}+\mathbf{1}$

8: until $(n<N) \&$ (any stopping criterion is not reached)

\section{2) Particle Swarm Optimization (PSO)}

It was proposed by [26], based on their study carried out about the social behavior of some animal groups in food searching activities. Unlike other evolutionary computing techniques, PSO is well-known cooperative and sharing information with neighboring particles. This global optimization heuristic method has been considered into a vast number of applications [27]. Another important fact is related to the manner its particles' positions and velocities are updated via two basic but powerful equations. Then, the new position 
$\left(\vec{x}_{m}^{n+1} \in \mathfrak{x}^{n+1}\right)$ and velocity $\left(\vec{v}_{m}^{n+1} \in \mathfrak{V}^{n+1}\right)$ for the $m$-th particle are obtained with

$$
\vec{x}_{m}^{n+1}=\vec{x}_{m}^{n}+\vec{v}_{m}^{n+1}
$$

and,

$$
\vec{v}_{m}^{n+1}=w \vec{v}_{m}^{n}+\phi_{1} \vec{r}_{1} \odot\left(\vec{x}_{m, *}^{n}-\vec{x}_{m}^{n}\right)+\phi_{2} \vec{r}_{2} \odot\left(\vec{x}_{*}^{n}-\vec{x}_{m}^{n}\right)
$$

where $\omega \in(0,1]$ is the inertial factor; $\phi_{1}$ and $\phi_{2}$ are the self and swarm confidence coefficients, respectively; $\vec{r}_{1}$ and $\vec{r}_{2}$ are vectors of i.i.d. random variables with $U(0,1)$; and $\vec{x}_{*}^{n}$ and $\vec{x}_{m, *}^{n}$ are the best position found by the entire swarm or population and by the $m$-th particle, respectively. Consequently, it is possible to describe the iterative procedure which forges the PSO method as Pseudocode 3 shown

PSEudocode 3. Particle Swarm Optimization (PSO).

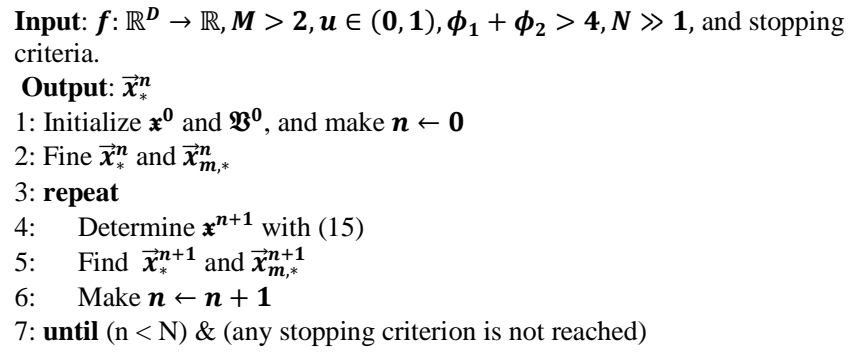

\section{Methodology}

For all simulations we used an Apple MacBook Pro computer and the commercial numerical platform Mathworks Matlab. Pseudocode 1 was implemented to solve (8) by employing Genetic Algorithm (GA) and Particle Swarm Optimization (PSO). To avoid unpractical solutions, the inequality constraints for string's currents $\left(I_{s t r, k} \geq 0\right)$ were considered into a modified version of (14) with an added penalty function [28]. The resulting objective function to be minimized was

$$
f_{o b j}(\vec{x}):=\sum_{k=1}^{M}\left[\left(F_{k}(\vec{x})\right)^{2}+\left(\max \left\{0,-I_{s t r, k}(\vec{x})\right\}\right)^{2}\right] \text {. }
$$

Tune parameters employed for GA were $M=50 D, M e=2$ and $C_{f}=0.8$, and for PSO were $M=\min \{100,10 D\}, \varphi_{1}=$ $\varphi_{2}=1.49$ and $\omega \in[0.1,1.1]$. Furthermore, three demonstrative examples, varying the number of modules and irradiance distribution, were carried out.

In this article, the PV panel considered for the simulated generator was the ERDM 85, which is formed by one module of 36 cells connected in series $\left(N_{s}=36\right)$. The generator's main electrical characteristics are: $I_{S C, S T C}=5.13 \mathrm{~A}, V_{o c, S T C}=$ $21.78 \mathrm{~V}, \quad I_{m p p, S T C}=4.8 \mathrm{~A}, \quad V_{m p p, S T C}=17.95 \mathrm{~V}, \quad \alpha_{I s c}=$ $0.02 \% / \mathrm{K}, \alpha_{V o c}=-0.37 \% / \mathrm{K}$, and $N s=36$; where the subindex $S T C$ indicates the Standard Test Conditions, $I_{S C, S T C}$ and $I_{m p p, S T C}$ are the short-circuit and MPP currents, respectively, $V_{o c, S T C}$ and $V_{m p p, S T C}$ are the open-circuit and MPP voltages, and, finally, $\alpha_{I S c}$ and $\alpha_{V o c}$ are the temperature coefficients of $I_{S c}$ and $V_{o c}$, respectively.

From the datasheet's parameters it is possible to estimate the single-diode model parameters in STC using the method proposed by [17], and employing the adjustments proposed by [29]. The obtained parameters were: $I_{p h}=5.133 \mathrm{~A}, \eta=$ $1.061, I_{\text {sat }}=1.184 \eta \mathrm{A}, R_{s}=0.186 \Omega$, and $R_{h}=261.099 \Omega$. Moreover, the bypass diode's parameters were calculated by using two points from the Microsemi SFDS1045Le3's datasheet [30], obtaining $I_{s a t, b d}=85.154 \mathrm{~mA}$ and $\eta_{b d}=$ 1.634. Both, the module's and bypass diode's parameters were considered the same for all the modules, with the exception of $I_{p h}$, since it is proportional to the irradiance of each module.

Partial shading conditions for each string were introduced by defining $I_{p h}$ per module, as shown

$$
\left[\begin{array}{c}
I_{p h, 1} \\
I_{p h, 2} \\
\vdots \\
I_{p h, N}
\end{array}\right]=I_{p h}\left[\begin{array}{c}
P_{G, 1} \\
P_{G, 2} \\
\vdots \\
P_{G, N}
\end{array}\right]=I_{p h} \vec{P}_{G}
$$

where $I_{p h, i}$ represents the photovoltaic current of module $i$, $P_{G, i}$ is the irradiance's proportion in STC $\left(G_{S T C}=\right.$ $1000 \mathrm{~W} / \mathrm{m}^{2}$ ) over module $i$ and $I_{p h}$ was defined in the previous paragraph. Finally, the search range for solving the simulated examples was stated in accordance to the datasheet's information as follows: $\left[-0.45, V_{\text {oc STC }}\right]$ for each module's voltage and $[-0.45,0]$ for the blocking diodes' voltage.

\section{RESULTS AND DISCUSSION}

In this section we include the solution of the model described above using GA and PSO, and the solution of the Equivalent Circuit Model, which was taken as a reference.

Fig. 3 shows the simulation results for the case of three modules where the irradiance (solar irradiation) with $\vec{P}_{G}=$ $[0.9,0.6,0.1]^{\top}$. This figure plots the array current as a function of the array voltage. We use the solution of the equivalent circuit model as a reference. As observed, both algorithms attained the exact solution. Furthermore, Fig. 4 shows the array power as a function of the array voltage. The results are identical to those of the equivalent circuit. If we compare the solution of the two algorithms, we can observe that PSO seems to be more accurate than GA, for the present case (Fig. $5)$.

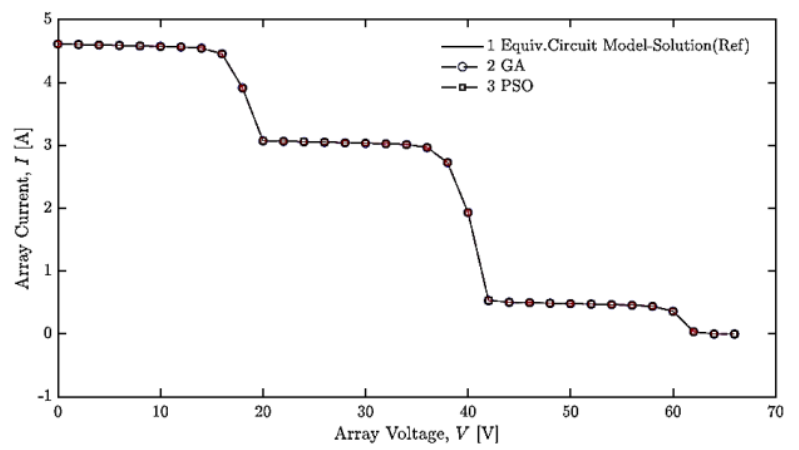

Fig. 3. Current vs. Voltage curve for the three modules array.

Now, we simulate an array made of eight modules receiving them half of $G_{S T C}$, i.e., under homogeneous conditions; therefore, $\vec{P}_{G}$ is a vector of eight elements, where all of them 
are 0.5. Again, both algorithms were capable to get the same solution (ideal) given by solving the equivalent circuit model. The corresponding I-V and P-V curves for the array are introduced in Fig. 6 and Fig. 7, respectively. Those curves describe the expected behavior for the two algorithms and the error seems negligible for the current and power calculation.

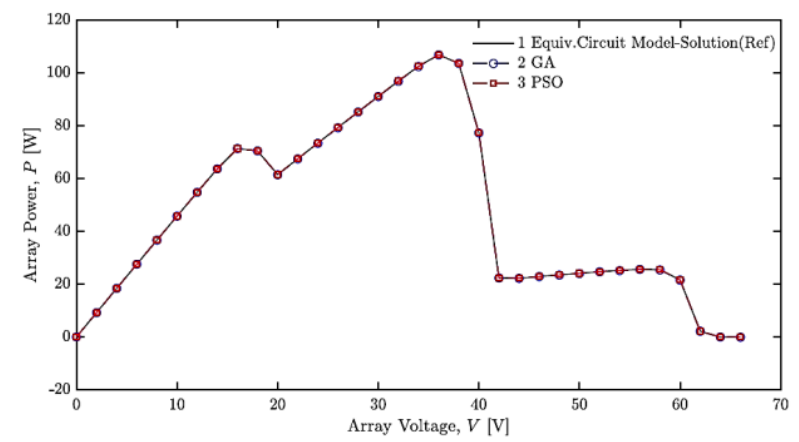

Fig. 4. Power vs. voltage curve for the three modules array.

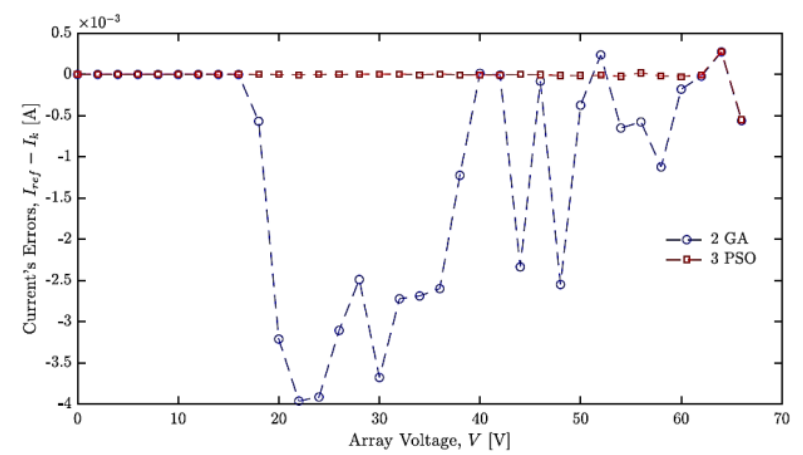

Figure 5. Currents' Errors $\left(I_{r e f}-I_{k}\right)$ for the three modules array.

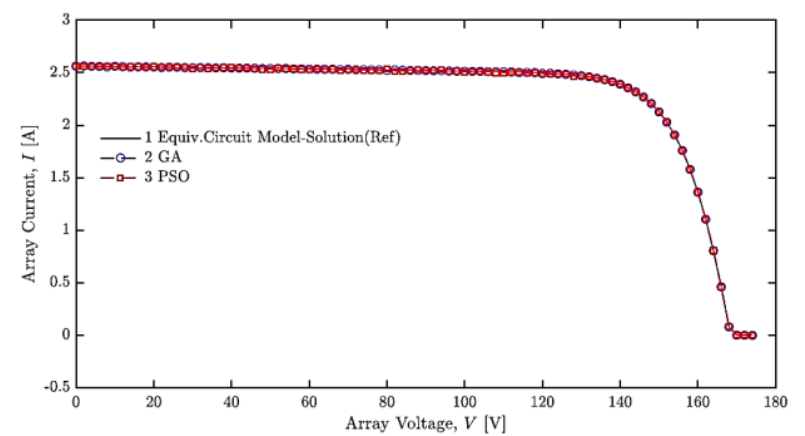

Fig. 6. Current vs. Voltage curve for the eight modules array with homogeneous conditions.

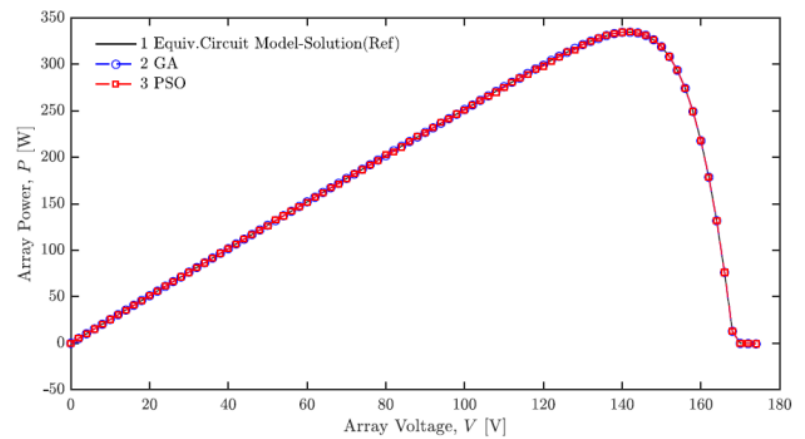

Fig. 7. Power vs. Voltage curve for the eight modules array with homogeneous conditions.
In order to compare the solution methods, we use three parameters: the computational time, the RMSE (Root Mean Square Error) and the number of times that the objective function is called ( $\mathrm{F}$ eval.), as shown in Table I. The RMSE compares a predicted value (from the two algorithms) and a known value (from the solution of the equivalent circuit model). As can be observed, the two population-based algorithms used much more time to obtain the solution than the reference model, but the final results are quite similar to those expected (indicated by the low values for the RMSE). The same can be concluded for the case of the number of times the objective function is called (F eval.). Nonetheless, PSO resulted to be more efficient than GA.

$$
\text { TABLE I. }
$$

MAIN SIMULATION RESULTS FOR THE CASE OF AN ARRAY MADE OF EIGHT

\begin{tabular}{cccc}
\multicolumn{4}{c}{ MODULES WITH HOMOGENEOUS CONDITIONS } \\
\hline \hline Method & Time [s] & RMSE & F eval. \\
\hline Reference & 0.559 & - & - \\
GA & 545.1 & 0.0015737 & 9510800 \\
PSO & 80.26 & 0.0050436 & 1737800 \\
\hline \hline
\end{tabular}

It is worth noting that, the simulation of the equivalent circuit requires a circuit simulation software, where a modification in the PV generator size implies a modification and re-connection of the entire circuit. Moreover, the modification of the parameter values of all the modules may need manual modification per element in the circuit. All these modifications require an additional time which is not considered in the time reported in Table I, such a table only considers the time required to solve the equivalent circuit.

Having now an array under mismatching conditions, which is composed of four modules with an irradiation of $900 \mathrm{~W} / \mathrm{m}^{2}$ and another four with $500 \mathrm{~W} / \mathrm{m}^{2} \quad\left(\vec{P}_{G}=\right.$ $\left.[0.9,0.5,0.5,0.9,0.5,0.9,0.5,0.9]^{\top}\right)$, their I-V and P-V curves are shown in Fig. 8 and Fig. 9, respectively. For this large PV system, both algorithms also reached the expected solution. Both its form and values are the same as those obtained by solving the equivalent circuit model.

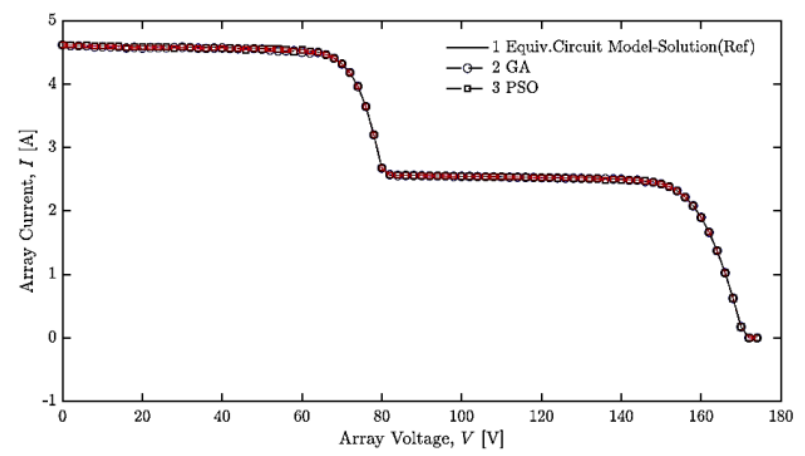

Fig. 8. Current vs. Voltage curve for the eight modules array with partial shading conditions. 


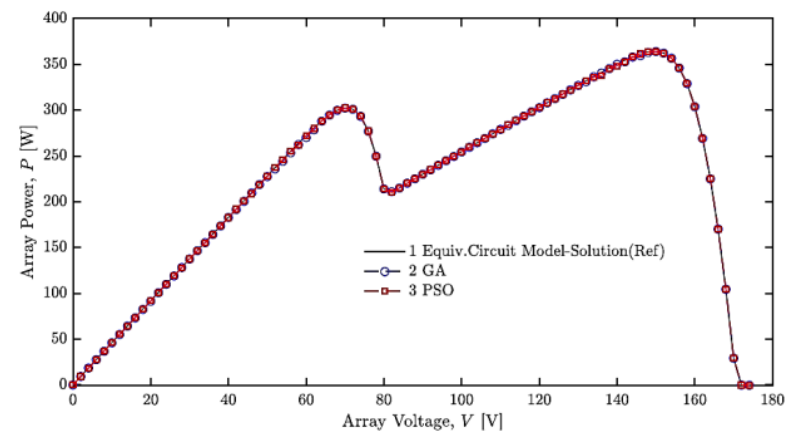

Fig. 9. Power vs. Voltage curve for the eight modules array with partial shading conditions.

In Table II, we observe the same trend as before. The computation time of both algorithms is substantially longer to achieve the desired response. Nevertheless, their responses are good enough for any further calculation required. PSO was again more efficient than GA according to the number of evaluations of the objective function (i.e., column $\mathrm{F}$ eval.) and the calculation time. There were also a large number of simulations that generated results close to the solution but not identical. Therefore, it is important to bear in mind that they are algorithms that depend both on the number of particles used to find the global optimum, and on the parameters of adjustment. The greater the number of these, the longer the calculation time will be, and the accuracy will probably be better.

TABLE II.

MAIN SIMULATION RESULTS FOR THE CASE OF AN ARRAY MADE OF EIGHT MODULES WITH MISMATCHING CONDITIONS.

\begin{tabular}{cccc}
\hline \hline Method & Time [s] & RMSE & F eval. \\
\hline Reference & 1.5659 & - & - \\
GA & 1677.4 & 0.012173 & 26453000 \\
PSO & 231.90 & 0.0088845 & 4806000 \\
\hline \hline
\end{tabular}

\section{CONCLUSIONS}

In this article, we described the main components of a photovoltaic generator system given emphasis to its mathematical representation. As stated, accurate mathematical models of PV arrays operating under uniform and mismatching conditions are important for different applications in PV systems. They can be used to estimate the power and energy produced by a PV generator, to perform realistic economic analysis PV systems, and many more practical applications. Here we proposed a numerical strategy based on the transformation of the problem into an optimization one. After the setting-up of the objective function, we solved it through two traditional global optimization algorithms, the Genetic Algorithm, and the Particle Swarm Optimization. As shown in the results section, both of them were capable to solve the problem. It was observed that the main drawback is their high computation time, but it can be alleviated, probably, by optimizing their codes or using a different programming language like $\mathrm{C}$ or $\mathrm{C}++$. Nevertheless, it should be an interesting alternative when dealing with larger arrays.
Finally, one of the main advantages of the proposed method is that it does not require a guess solution close to the real one, like Newton-Raphson, damped Newton-Raphson methods or other conventional numerical methods to solve nonlinear equation systems. In fact, this method can be extended to other configurations of PV generators, e.g., totalcross tied, bridge-linked or honey-comb.

\section{REFERENCES}

H. Häberlin, Photovoltaics: System Design and Practice. Chichester, UK: John Wiley \& Sons, Ltd, 2012. DOI:10.1002/9781119976998

[2] G. Petrone, C. A. Ramos-Paja, and G. Spagnuolo, Photovoltaic Sources Modeling. Chichester, UK: John Wiley \& Sons, Ltd, 2017. DOI: $10.1002 / 9781118755877$

[3] IEA, “2018 Snapshot of Global Photovoltaic Markets,” 2018.

[4] REN21, "Renewables 2018 Global Status Report," Paris, 2018.

[5] T. Ma, H. Yang, and L. Lu, "Solar photovoltaic system modeling and performance prediction," Renew. Sustain. Energy Rev., vol. 36, pp. 304-315, 2014. DOI:10.1016/j.rser.2014.04.057

[6] D. Jena and V. V. Ramana, "Modeling of photovoltaic system for uniform and non-uniform irradiance: A critical review," Renew. Sustain. Energy Rev., vol. 52, pp. 400-417, 2015. DOI:10.1016/j.rser.2015.07.079

[7] R. Ramaprabha and B. L. Mathur, "A Comprehensive Review and Analysis of Solar Photovoltaic Array Configurations under Partial Shaded Conditions," Int. J. Photoenergy, vol. 2012, pp. 1-16, 2012. DOI:10.1155/2012/120214

[8] N. Femia, G. Petrone, G. Spagnuolo, and M. Vitelli, Power Electronics and Control Techniques for Maximum Energy Harvesting in Photovoltaic Systems, 1st Ed. CRC Press 2012, 2012. DOI:10.1201/b14303

G. Petrone, G. Spagnuolo, and M. Vitelli, "Analytical model of mismatched photovoltaic fields by means of Lambert W-function," Sol. Energy Mater. Sol. Cells, vol. 91, no. 18, pp. 1652-1657, Nov. 2007. DOI:10.1016/j.solmat.2007.05.021

[10] G. Petrone and C. a. Ramos-Paja, "Modeling of photovoltaic fields in mismatched conditions for energy yield evaluations," Electr. Power Syst. Res., vol. 81, no. 4, pp. 1003-1013, Apr. 2011. DOI:10.1016/j.epsr.2010.12.008

[11] M. L. Orozco-Gutierrez, J. M. Ramirez-Scarpetta, G. Spagnuolo, and C. a. Ramos-Paja, "A technique for mismatched PV array simulation," Renew. Energy, vol. 55, pp. 417-427, Jul. 2013. DOI:10.1016/j.renene.2013.01.009

[12] E. Karatepe, M. Boztepe, and M. Çolak, "Development of a suitable model for characterizing photovoltaic arrays with shaded solar cells," Sol. Energy, vol. 81, no. 8, pp. 977-992, Aug. 2007. DOI:10.1016/j.solener.2006.12.001

[13] H. Patel and V. Agarwal, "Maximum Power Point Tracking Scheme for PV Systems Operating Under Partially Shaded Conditions," IEEE Trans. Ind. Electron., vol. 55, no. 4, pp. 1689-1698, Apr. 2008. DOI:10.1109/TIE.2008.917118

[14] H. Patel and V. Agarwal, "MATLAB-Based Modeling to Study the Effects of Partial Shading on PV Array Characteristics," IEEE Trans. Energy Convers., vol. 23, no. 1, pp. 302-310, Mar. 2008. DOI:10.1109/TEC.2007.914308

[15] A. Bassam, O. May Tzuc, M. A. Escalante Soberanis, L. J. Ricalde, and B. Cruz, "Temperature Estimation for Photovoltaic Array Using an Adaptive Neuro Fuzzy Inference System," Sustainability, vol. 9, no. 8, p. 1399, 2017. DOI:10.3390/su9081399

[16] D. Gonzalez Montoya, J. D. Bastidas-Rodriguez, L. A. TrejosGrisales, C. A. Ramos-Paja, G. Petrone, and G. Spagnuolo, "A 
Procedure for Modeling Photovoltaic Arrays under Any Configuration and Shading Conditions," Energies, vol. 11, no. 4, pp. 767-783, 2018. DOI:10.3390/en11040767

[17] J. Accarino, G. Petrone, C. a. Ramos-Paja, and G. Spagnuolo, "Symbolic algebra for the calculation of the series and parallel resistances in PV module model," in 2013 International Conference on Clean Electrical Power (ICCEP), 2013, pp. 62-66. DOI:10.1109/ICCEP.2013.6586967

[18] M. G. Villalva, J. R. Gazoli, and E. R. Filho, "Comprehensive Approach to Modeling and Simulation of Photovoltaic Arrays," IEEE Trans. Power Electron., vol. 24, no. 5, pp. 1198-1208, May 2009. DOI:10.1109/TPEL.2009.2013862

[19] J. D. Bastidas, E. Franco, G. Petrone, C. A. Ramos-Paja, and G. Spagnuolo, "A model of photovoltaic fields in mismatching conditions featuring an improved calculation speed," Electr. Power Syst. Res., vol. 96, pp. 81-90, Mar. 2013. DOI:10.1016/j.epsr.2012.10.020

[20] J. D. Bastidas-Rodriguez, D. Gonzalez, and C. A. Ramos-paja, "Model of Series-Parallel Photovoltaic Arrays Designed for Parallel Computing," in 2017 14th International Conference on Synthesis, Modeling, Analysis and Simulation Methods and Applications to Circuit Design (SMACD), 2017, pp. 0-3. DOI:10.1109/SMACD.2017.7981592

[21] D. Whitley, "A genetic algorithm tutorial," Stat. Comput., vol. 4, no. 2, pp. 65-85, 1994. DOI:10.1007/BF00175354

[22] A. K. Kar, "Bio inspired computing - A review of algorithms and scope of applications," Expert Syst. Appl., vol. 59, pp. 20-32, 2016. DOI:10.1016/j.eswa.2016.04.018

[23] C. W. Ahn, "Practical Genetic Algorithms," in Advances in Evolutionary Algorithms. Studies in Computational Intelligence, Vol 18., Berlin: Springer, 2006. DOI:10.1007/3-540-31759-7_2

[24] J. E. Baker, "Reducing Bias and Inefficiency in the Selection Algorithm," in Proceedings of the Second International Conference on Genetic Algorithms on Genetic Algorithms and Their Application, 1987, pp. 14-21.

[25] D. E. Goldberg, Genetic Algorithms in Search, Optimization and Machine Learning, 1st ed. Boston, MA, USA: Addison-Wesley Longman Publishing Co., Inc., 1989.

[26] J. Kennedy and R. Eberhart, "Particle swarm optimization," in Proceedings of ICNN'95 - International Conference on Neural Networks, 1995, vol. 4, pp. 1942-1948 vol.4. DOI:10.1109/ICNN.1995.488968

[27] Y. Zhang, S. Wang, and G. Ji, "A Comprehensive Survey on Particle Swarm Optimization Algorithm and Its Applications," Math. Probl. Eng., vol. 2015, 2015. DOI:10.1155/2015/931256

[28] S. S. Rao, Engineering Optimization: Theory and Practice, Fourth Edi. John Wiley \& Sons, Inc., 2009. DOI:10.1002/9780470549124

[29] W. De Soto, S. A. Klein, and W. A. Beckman, "Improvement and validation of a model for photovoltaic array performance," Sol. Energy, vol. 80, no. 1, pp. 78-88, Jan. 2006. DOI:10.1016/j.solener.2005.06.010

[30] Microsemi, "SFDS1045Le3 Photovoltaic Bypass Diodes." pp. 1-5, 2018.

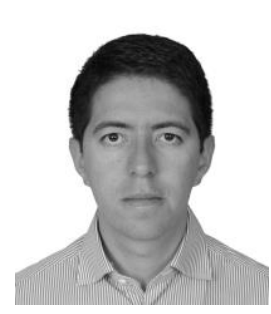

Juan David Bastidas-Rodriguez was born in Pasto, Nariño (Colombia) in 1985. He received the B.Sc degree in electronics from the Universidad del Valle, Cali, Colombia, in 2008, and the Ph.D. degree from the Universidad del Valle and the University of Salerno, Salerno, Italy, in
2014 and 2015, respectively.

He was Assistant professor at Universidad Industrial de Santander, Bucaramanga, Colombia, from 2015 to 2018 and since 2019, he has been an Associate Professor at the Department of Electrical, Electronic and Computation Engineering with the Universidad Nacional de Colombia, Manizales, Colombia. His main research interests include modeling, control, sizing, and diagnosis of renewable energy systems.

ORCID: http://orcid.org/0000-0002-4634-2642

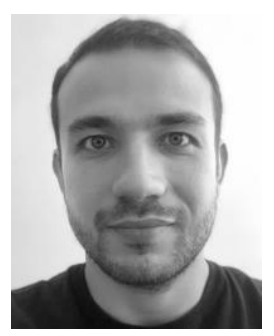

Jorge Mario Cruz-Duarte (M'15) was born in Ocaña, Norte de Santander (Colombia) in 1990. He received the B.Sc. and M.Sc. degrees in electronics engineering from Universidad Industrial de Santander, Bucaramanga, Colombia, in 2012 and 2015, respectively, and the Ph.D. in electrical engineering from Universidad de Guanajuato, Salamanca,

México, in 2018.

Since 2019, he is a Postdoctoral Fellow at the Grupo de Investigación con Enfoque Estratégico en Sistemas Inteligentes, at Tecnológico de Monterrey, Monterrey, México. His research interests include data science, optimization, mathematical methods, digital signal processing, thermodynamics, and fractional calculus.

ORCID: http://orcid.org/0000-0003-4494-7864

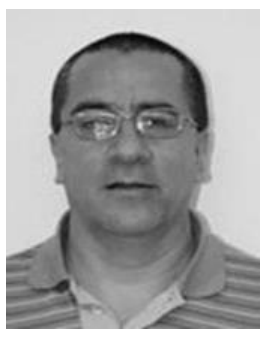

Carlos Rodrigo Correa Cely received the B.E. degree in chemical engineering from the Universidad Nacional de Colombia, Bogotá, Colombia, the M.Sc. degree in chemical engineering from the Universidad Industrial de Santander, Bucaramanga, Colombia, and the M.Sc. and Ph.D. degrees in polymer science and engineering from Lehigh University,

Bethlehem, PA, USA.

He is currently a full-time Professor with the School of Electrical, Electronic and Telecommunication Engineering, Universidad Industrial de Santander, Bucaramanga. His current research interests include microwave heating, global optimization, heat transfer, and polymers.

ORCID: http://orcid.org/0000-0002-6507-1809 